Aileen Roman, MD; Hans P. Van Lancker, MD, FRCSC

Department of Family Medicine, Boston University School of Medicine (Dr. Roman); Department of Orthopedics, St. Elizabeth's Medical Center and Harvard Medical School, Boston (Dr. Van Lancker)

- aileen.roman@bmc.org

Dr. Roman reported no potential conflict of interest relevant to this article. Dr Van Lancker is a consultant to Conventus Orthopaedics, Globus Medical, and Stryker.

\title{
Painful foot or ankle? Don't overlook these 5 injuries
}

\author{
A missed diagnosis of one of these conditions risks \\ delay in referral for orthopedic evaluation and surgical \\ management-possibly leading to complications.
}

\section{PRACTICE RECOMMENDATIONS \\ $\mathrm{F}$}

> Suspect higher-grade syndesmotic disruption (which typically requires surgical intervention) in patients whose ankle pain persists after 3 weeks of immobilization or who have a tibial or fibular diastasis on a plain film. (C)

> Order weight-bearing $x$-rays to make an accurate diagnosis of Lisfranc injury. Refer for potential surgical intervention if diastasis is evident at the base between the first and second metatarsals. (C)

> Distinguish between proximal diaphysial (Jones) fracture of the fifth metatarsal, diaphysial stress fracture, and avulsion fracture-essential because avulsion fracture can be treated nonoperatively but the other 2 require surgical intervention. (C)

Strength of recommendation (SOR)

A Good-quality patient-oriented evidence

B Inconsistent or limited-quality patient-oriented evidence

C Consensus, usual practice, opinion, disease-oriented evidence, case series oot and ankle injuries are among the most common conditions evaluated at primary care visits; the differential diagnosis of such injury is broad. ${ }^{1}$ Although many of these injuries are easily identified on imaging studies, a number of subtle, yet important, conditions can be easily missed, especially if you do not routinely encounter them. Given that broad differential, a high degree of suspicion is required to make an accurate diagnosis, which allows appropriate treatment within a reasonable time frame and minimizes the risk of long-term morbidity.

This article outlines the diagnosis and initial management of 5 important, yet often elusive, types of foot and ankle conditions: Achilles tendon rupture, injury to the syndesmosis, ankle fracture, Lisfranc injury, and proximal fracture of the fifth metatarsal.

\section{Achilles tendon rupture}

The Achilles tendon is the most frequently ruptured tendon in the body (approximately $20 \%$ of all large-tendon injuries) ${ }^{2}$; as many as $25 \%$ of cases are initially misdiagnosed. ${ }^{3}$

I Presentation. Patients frequently present with pain at the Achilles tendon -2 to $6 \mathrm{~cm}$ above the insertion into the calcaneus-and an inability to fully bear weight. ${ }^{4,5} \mathrm{~A}$ small percentage of patients are able to ambulate on the affected side, albeit with minor pain, which likely contributes to the rate of missed diagnosis. Absence of difficulty bearing weight is due to the presence of secondary plantar flexors, which can compensate for loss of chief plantar flexor function by the Achilles tendon. ${ }^{2}$

I Examination of a patient with an Achilles tendon rupture typically reveals edema, bruising, and a palpable gap within the tendon, 2 to $6 \mathrm{~cm}$ proximal to insertion. ${ }^{3,4}$ The Thompson test-squeezing the calf with the patient prone and the knee on the affected side flexed-can aid in diagnosis. When the Achilles tendon is intact, plantar flexion occurs at the ankle; 


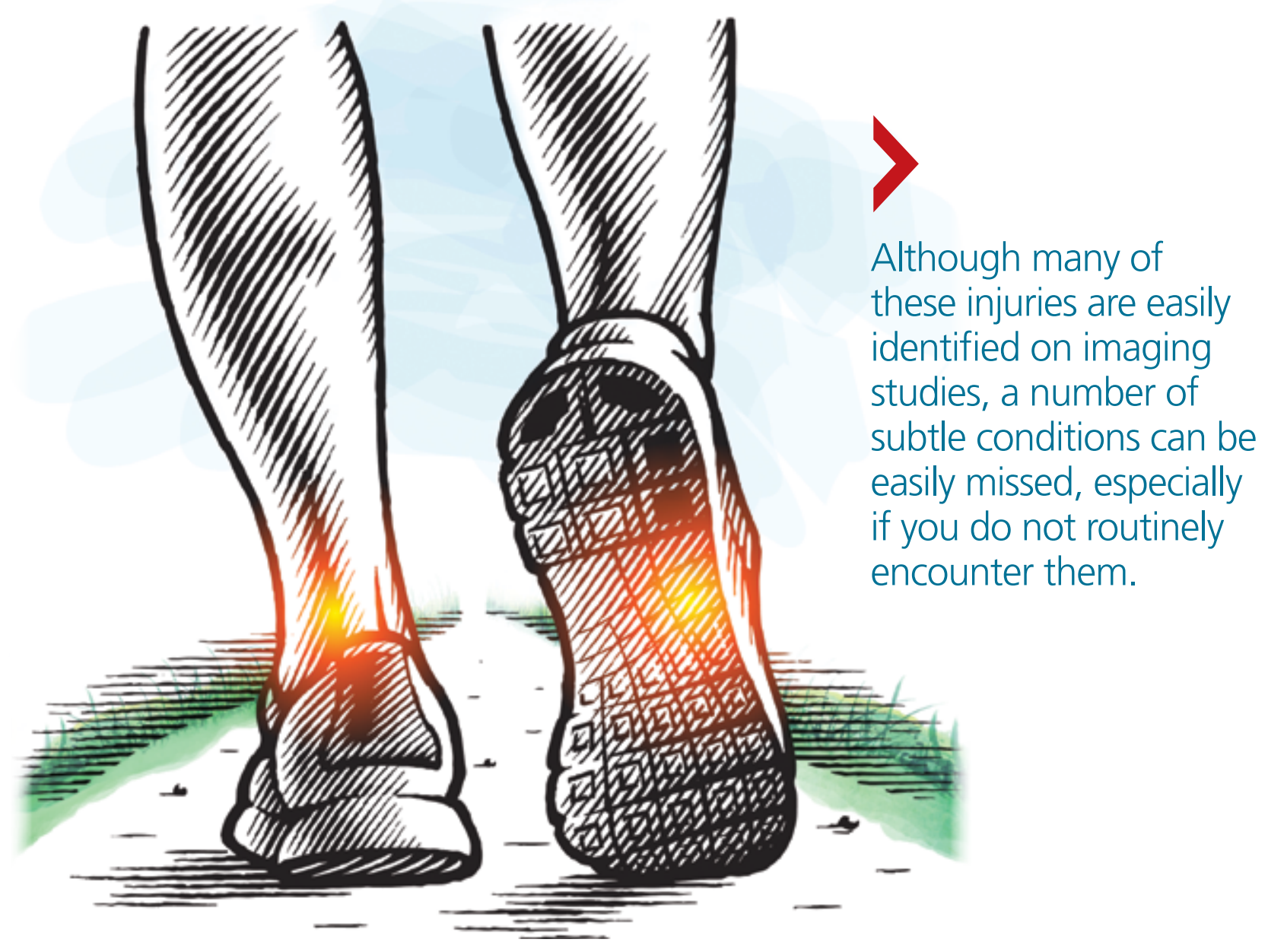

when the tendon is ruptured, plantar flexion is absent. ${ }^{5}$ The test can be modified when examining a patient who is unable to lie prone by having them rest the flexed knee on a chair while standing on the unaffected leg.

A diagnosis of Achilles tendon rupture is supported when at least 2 of the following conditions are met $^{4,5}$ :

- positive Thompson test

- decreased strength during plantar flexion of the ankle

- palpable gap or pain at the typical location (2-6 cm above insertion)

- increased passive ankle dorsiflexion upon gentle ranging of the ankle joint.

I Imaging has a limited role in the diagnosis of Achilles tendon rupture; because the findings of the physical examination are reliable, seserve $\mathrm{x}$-rays for cases in which the diagnosis remains uncertain after examination. ${ }^{2}$ Consid$\stackrel{\oplus}{\circ}$ er ordering plain x-rays to rule out an avulsion 硋 fracture at the insertion of the Achilles tendon; ultrasonography or magnetic resonance imag坚 ing (MRI) might assist you in detecting the rup- ture proper, along with the location of the tear for surgical planning, if surgery is deemed necessary by an orthopedic surgeon. ${ }^{3-5}$

Management. Some degree of controversy surrounds preferred treatment of Achilles tendon rupture, although available evidence demonstrates that these injuries can be effectively managed by surgical repair or nonoperative treatment, as outcomes are comparable. ${ }^{3,5}$ Operative management tends to reduce the risk of repeat rupture, compared to nonoperative treatment; however, the potential for surgical complications, including wound infection, sensory disturbance, and adhesions favors nonoperative treatment. , $^{3,46}$

Nonoperative treatment consists of referral to a functional rehabilitation program, without which outcomes are, on the whole, less favorable than with surgery. ${ }^{3,6}$ Surgery is preferred if functional rehabilitation is unavailable, 6 months of conservative management fails, or there is avulsion injury.,4,6

Injury to the syndesmosis

A complex of ligaments that provide dynamic 


\section{$>$}

Some patients with Achilles tendon rupture can walk on the affected side, even with minor pain; the diagnosis might be missed without further in-depth evaluation. stability to the ankle joint, the tibiofibular syndesmosis comprises:

- the anterior inferior tibiofibular ligament

- the posterior inferior tibiofibular ligament

- the inferior transverse tibiofibular ligament

- the interosseous membrane.

These structures are further supported by the deltoid ligament. ${ }^{7,8}$

Commonly referred to as a "high ankle sprain," a syndesmotic injury is present in as many as $20 \%$ of ankle fractures and $5 \%$ to $10 \%$ of ankle sprains. Injury typically results from external rotation with hyperdorsiflexion of the ankle. Recovery is typically prolonged (ie, twice as long as recovery from a lateral ankle sprain). The diagnosis is missed in as many as $20 \%$ of patients; failure to recognize and treat syndesmotic instability appropriately can lead to posttraumatic arthritis. ${ }^{7,9}$

I Presentation. Patients generally present with ankle pain, swelling, instability, pain when walking on uneven terrain, and pain upon push-off. ${ }^{9}$

IExamination reveals reduced passive ankle dorsiflexion and tenderness upon palpation of individual ligaments. Several clinical tests have been described to aid in detecting this often-elusive diagnosis ${ }^{7,9,10,11}$ :

- Squeeze test. The patient sits with the knee on the affected side bent at a $90^{\circ}$ degree angle while the examiner applies compression, with one or both hands, to the tibia and fibula at midcalf. The test is positive when pain is elicited at the level of the syndesmosis just above the ankle joint. ${ }^{9,11}$

- External rotation test. External rotation of the foot and ankle relative to the tibia reproduces pain.

- Crossed leg test. The affected ankle is crossed over the opposite knee in a figure- 4 position. The test is positive when pain is elicited at the syndesmosis. ${ }^{10}$

- Cotton test. The proximal lower leg is steadied with 1 hand and the plantar heel grasped with the other hand. Pain when the heel is externally rotated (and radiographic widening of the syndesmosis under fluoroscopy) signal syndesmotic instability.

- Fibular translation test. When anterior or posterior drawer force is applied to the fibula, pain and increased translation of the fibula (compared to the contralateral side) suggest instability.

With the Cotton and fibular translation tests, interexaminer technique is more variable and findings are less reproducible. ${ }^{8}$ Taken alone, none of the above-listed tests are diagnostic; they can, however, assist in making a diagnosis of an injury to the syndesmosis. ${ }^{11}$

I Imaging typically involves anteroposterior [AP], lateral, and mortise plain films of the ankle and weight-bearing AP and lateral views of the tibia and fibula. ${ }^{9}$ Important measures on weight-bearing AP $\mathrm{x}$-rays are the tibiofibular clear space (abnormal, $>6$ $\mathrm{mm}$ ) and the tibiofibular overlap (abnor$\mathrm{mal},<6 \mathrm{~mm}$ ) (both abnormalities shown in FIGURE 1). Comparing films of the affected ankle with views of the contralateral ankle is often useful.

I Management of syndesmotic injuries depends on degree of disruption:

- Grade 1 injury is a sprain without diastasis on imaging. Management is conservative, with immobilization in a splint or boot for 1 to 3 weeks, followed by functional rehabilitation over 3 to 6 weeks. $^{10}$

- Grade 2 injury is demonstrated by diastasis on a stress radiograph. Although evidence to guide successful identification of a grade 2 injury is lacking, it is clinically important to make that identification because these injuries might require surgical intervention, due to instability. Because the diagnosis of this injury can be challenging in primary care, high clinical suspicion of a grade 2 injury makes it appropriate to defer further evaluation to an orthopedic surgeon. On the other hand, if suspicion of a grade 2 injury is low, a trial of conservative management, with weekly clinical assessment, can be considered. A diagnosis of grade 2 injury can be inferred when a patient is unable to perform a singleleg hop after 3 weeks of immobiliza- 
tion; referral to an orthopedic surgeon is then indicated. ${ }^{12}$

- Grade 3 injury is frank separation at the distal tibiofibular joint that is detectable on a routine plain film. Management-surgical intervention to address instability-is often provided concurrently with the treatment for a Danis-Weber B or C fracture, which tends to coexist with grade 3 syndesmotic injury. (The Danis-Weber A-B$\mathrm{C}$ classification of lateral ankle fracture will be discussed in a bit.)

\section{Ankle fracture}

Fracture of the ankle joint is among the more common fractures in adults, comprising $10 \%$ of all fractures. ${ }^{13,14}$ The ankle joint is defined as the junction of 3 bony structures: (1) the distal ends of the tibia and fibula and (2) the trochlea of the talus, all stabilized by (3) the collateral ligament complex. Appropriate diagnosis and timely intervention are needed to prevent long-term posttraumatic joint degeneration.

I Presentation, examination, and imaging. In addition to difficulty bearing (or inability to bear) weight, patients with suspected ankle fracture can present with tenderness or pain, swelling (generally, the more severe the injury, the more severe the swelling, although this finding is time-dependent), and ecchymosis. However, distinguishing fracture from a ligamentous injury is often difficult by physical examination alone; the evidencebased Ottawa Ankle Rules can guide determination of the need for radiographic imaging, although this tool is less reliable in certain patient populations (TABLE ${ }^{15-17}$ ). ${ }^{13,15-17}$

I Management. A widely used classification system for guiding ankle fracture management is the Danis-Weber classification (FIGURE 2). In this scheme, type A fractures (distal to the level of the tibial plafond) are managed with ankle stabilization bracing without immobilization. Nondisplaced type $B$ and $C$ fractures (at the level of the tibial plafond and proximal to it, respectively) should be treated with 6 weeks of immobilization in a walking boot; close follow-up within 1 week of injury is recommended to ensure that no displacement of fragments has oc-

\section{FIGURE 1}

\section{Injury to the syndesmosis}

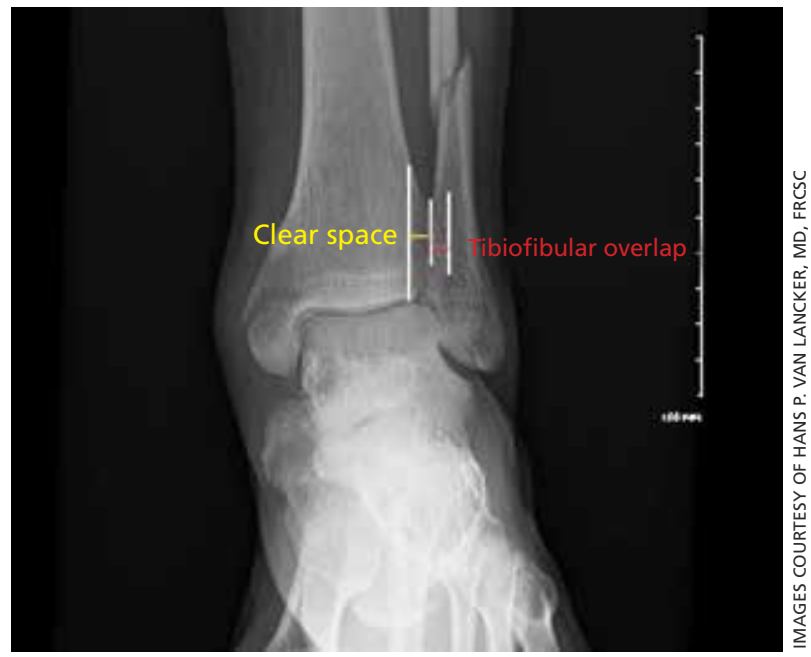

On a weight-bearing anteroposterior plain film of suspected syndesmosis injury, look for abnormalities, such as the ones shown here, of the tibiofibular clear space (ie, $>6 \mathrm{~mm}$ ) and the tibiofibular overlap (ie, $<6 \mathrm{~mm}$ ).

curred. Type B and C fractures need to be followed until bony union is achieved. If there is radiologic evidence of a fracture line after 3 months, referral to an orthopedic surgeon is indicated for management of delayed union.

Common indications for referral to Orthopedics for surgical intervention of ankle fracture include open fracture, bimalleolar and trimalleolar fracture, posterior malleolar fracture, medial malleolar displacement $>2 \mathrm{~mm}$, and lateral malleolar displacement $>3 \mathrm{~mm}^{18}$

Special concern: Talar fracture. Although talar fracture is rare, the injury is important to detect because a limited blood supply places fragments at risk of avascular necrosis. ${ }^{19}$ Talus fracture is frequently confused with ankle sprain because initial x-rays are not always revelatory. ${ }^{20} \mathrm{~A}$ high index of suspicion is required to make the diagnosis, which should be suspected in high-energy injuries that result in pain and swelling of the ankle accompanied by difficulty weightbearing, severely reduced range of motion, and tenderness to palpation at different areas of the talus. ${ }^{1}$ Computed tomography (CT) or MRI might be necessary to detect a talar fracture if initial $\mathrm{x}$-rays are negative. A low threshold for surgical management of talar fracture 
TABLE

Are x-rays needed to differentiate ankle injuries? Ottawa Ankle Rules ${ }^{\mathrm{a}}$ often provide an answer ${ }^{15-17}$

\begin{tabular}{|c|c|}
\hline An ankle series is indicated when: & A foot series is indicated when: \\
\hline There is pain in the malleolar zone & There is pain in the midfoot zone \\
\hline AND & AND \\
\hline $\begin{array}{l}\text { There is bone tenderness at the posterior edge or the tip of the } \\
\text { lateral or medial malleolus }\end{array}$ & $\begin{array}{l}\text { There is bone tenderness at the base of the fifth metatarsal or } \\
\text { at the navicular }\end{array}$ \\
\hline OR & OR \\
\hline $\begin{array}{l}\text { The patient is unable to bear weight immediately after injury } \\
\text { and for } 4 \text { steps in the emergency department or physician's } \\
\text { office }\end{array}$ & $\begin{array}{l}\text { The patient is unable to bear weight immediately after injury } \\
\text { and for } 4 \text { steps in the emergency department or physician's } \\
\text { office }\end{array}$ \\
\hline
\end{tabular}

a Ottawa Ankle Rules have not been validated in patients who are < 18 years, who are pregnant, or who have a neuropathic disorder or head injury. It is also inappropriate to use the Rules in patients whose injury occurred $>10$ days before presentation or who have an injury of the skin (burns, lacerations).

means that referral to Orthopedics is indicated once this injury is diagnosed. ${ }^{21}$

I Other frequently missed types of ankle fracture are shown in FIGURE $3 .^{22}$ These are relatively uncommon injuries that can be missed for a number of reasons, alone or in combination, including their subtlety on radiography, their often vague clinical presentation, and providers' lack of awareness of these types. Identification or strong suspicion of fracture at any of these sites (ie, in a patient who is persistently unable to bear weight) should prompt orthopedic referral.

\section{Lisfranc injury}

The tarsometatarsal joint comprises 3 cuneiforms, the cuboid, and 5 metatarsals. Stability is maintained by an intricate ligamentous complex. Lisfranc injury comprises a spectrum of midfoot injuries in which 1 or more metatarsals are displaced from the tarsus. These injuries are both rare and notoriously difficult to diagnose: As many as $20 \%$ of cases are missed on initial assessment. Without proper treatment, long-term disability and deformity, such as pes planus, can result. ${ }^{22-24}$ Lisfranc injuries typically result from a direct blow to the midfoot or excessive pronation or supination in a plantarflexed foot. ${ }^{23}$

I Presentation. A historical clue to Lisfranc injury is a report of pain while walking down stairs. Patients can present with pain, swelling, and tenderness to palpation over the dorsal aspect of the Lisfranc joint. Weight-bearing on the injured foot frequently cannot be tolerated but is occasionally possible in some patients, especially those who have diabetes or other baseline neuropathy. ${ }^{23}$

I Examination. Physical examination can also reveal plantar ecchymosis, which is considered pathognomonic. Another highly supportive maneuver is passive abduction and pronation of the forefoot, which can elicit pain. ${ }^{25,26}$

Imaging. Lisfranc injury can be diagnosed on weight-bearing $\mathrm{x}$-rays; as many as one-half of cases are missed when only nonweight-bearing films are obtained. If initial weight-bearing cannot be tolerated by the patient, another attempt at imaging can be made after 1 week of rest. ${ }^{24}$

Distance $>2 \mathrm{~mm}$ between the base of the first and second metatarsals (FIGURE 4) or an avulsion fracture at the medial base of the second metatarsal or distal lateral corner of the medial cuneiform (the "fleck sign") supports a disturbance of the Lisfranc joint complex. ${ }^{24}$ Imaging of the contralateral foot might highlight the injury in subtle cases, followed by CT when diagnostic uncertainty persists. ${ }^{24,25}$

I Management of Lisfranc injury depends on the stability of the joint complex. Stable injury without diastasis can be managed conservatively with immobilization in a short walker boot and limited weight-bearing for 2 weeks, followed by weight-bearing as 


\section{FIGURE 2}

\section{Danis-Weber radiographic classification of ankle fracture guides treatment}

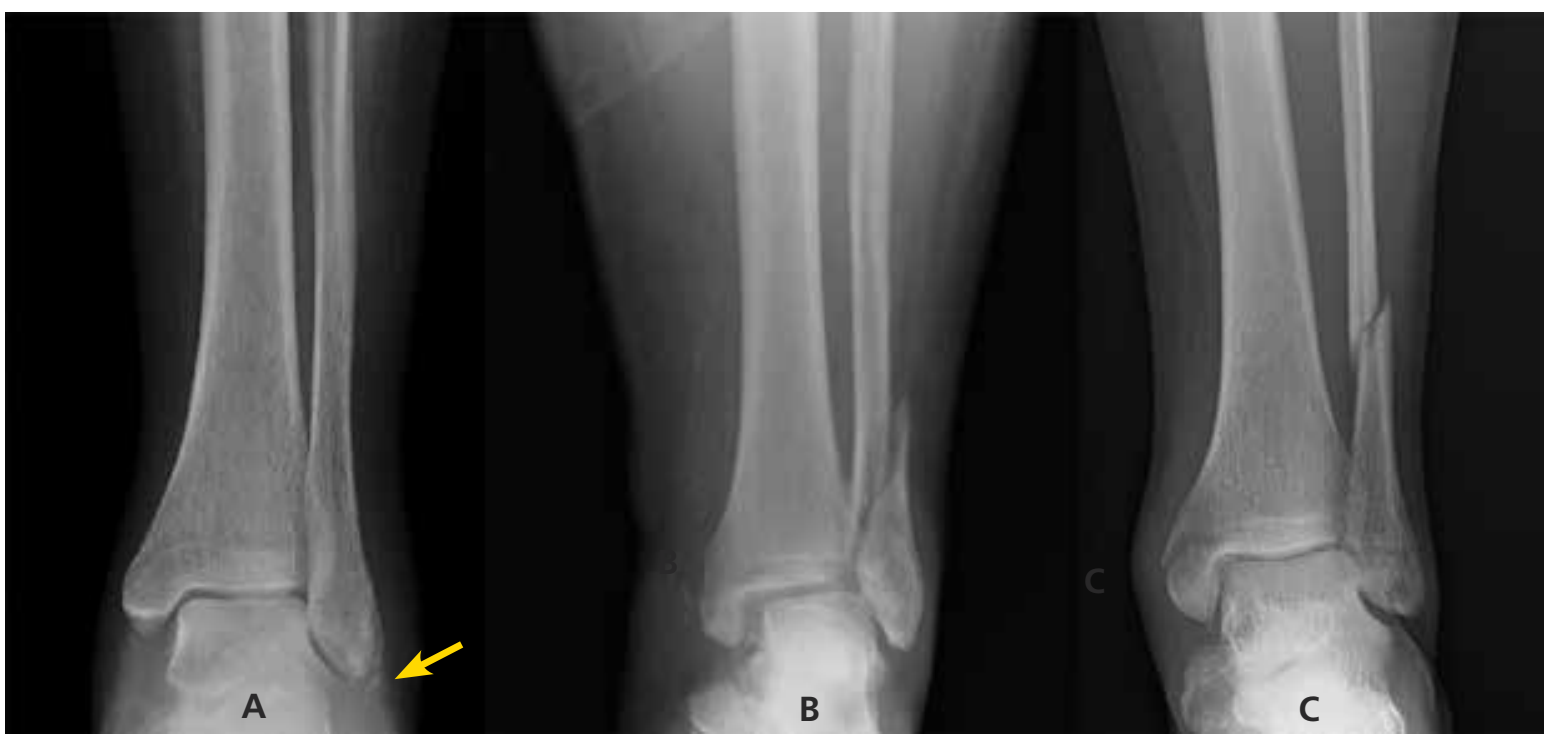

The Danis-Weber classification divides ankle fractures into 3 categories, based primarily on fibular involvement. Type A is a fracture below the tibial plafond. Type $\mathbf{B}$ is a fracture at the level of the tibial plafond, with the potential for associated syndesmotic injury. Type $C$ is a fracture of the fibula, above the tibial plafond, and there is likely associated syndesmotic injury. Note: The medial malleolus might be fractured and the deltoid ligament might be torn in any of the 3 fracture types $(A, B$, and $C)$.

Manage type $A$ fractures (A, arrow) with ankle stabilization bracing, without immobilization. Treat nondisplaced type B (B) and C fractures (C) with 6 weeks of immobilization in a walking boot.

\section{FIGURE 3}

Be alert for easily missed ankle fractures at these sites

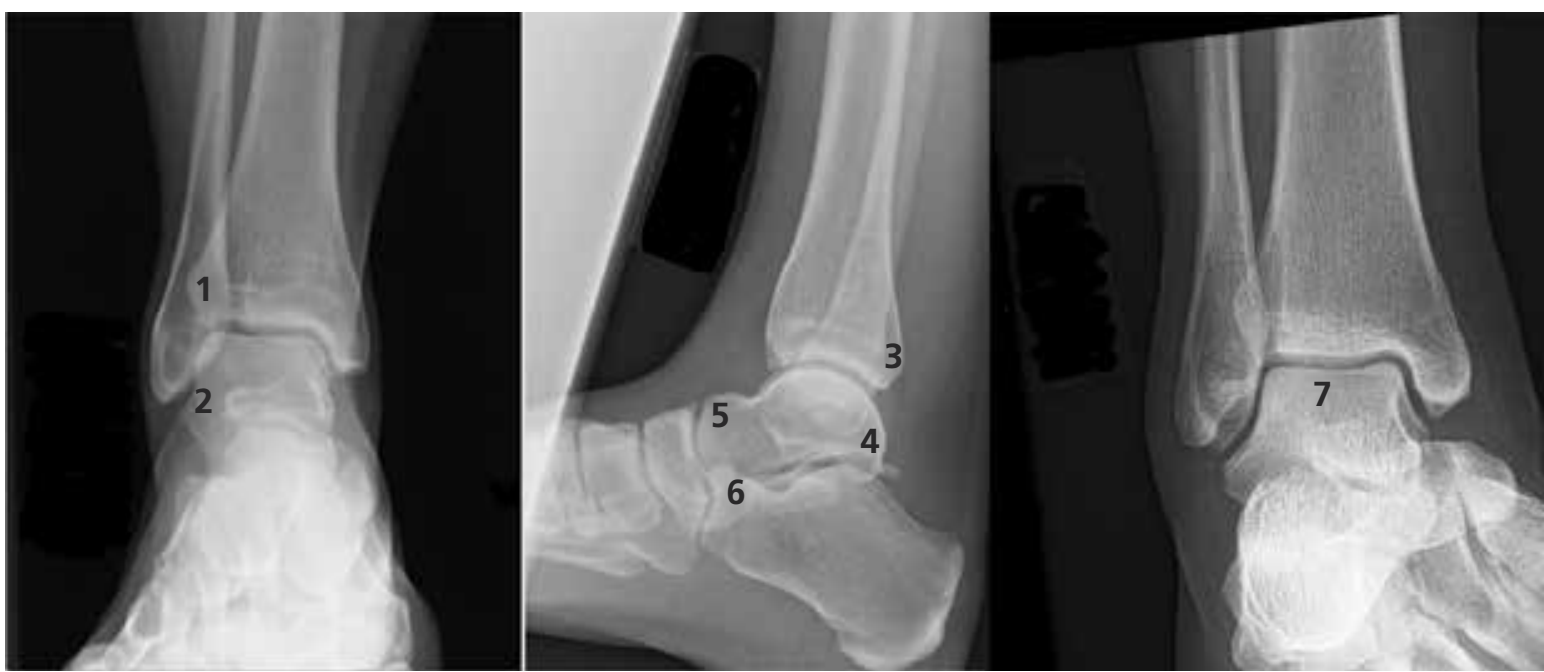

It's easy to miss fractures at these 7 sites: (1) anterior tibial tubercle, (2) lateral talar process, (3) posterior tibial malleolus, (4) posterior talus process, (5) dorsal to talonavicular joint, (6) anterior calcaneus process, and (7) talar dome.

tolerated in the boot if tenderness has improved. ${ }^{24}$ After 6 to 8 weeks, if the patient is pain-free with abduction stress, weight- bearing without the boot (but with a rigidsole shoe) is permissible for an additional 6 months. Sport-specific rehabilitation for an 


\section{Whether a syndesmotic injury is managed conservatively (immobilization, rehabilitation) or surgically depends on the degree (grade 1,2 , or 3) of disruption.}

\section{FIGURE 4}

\section{Clues to a disturbance of the Lisfranc joint complex}

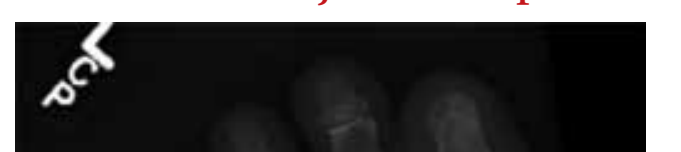

\section{Proximal fifth-metatarsal fractures}

These common fractures are classified in 3 broad categories: tuberosity avulsion fracture, proximal diaphyseal (Jones) fracture, and stress fractures of the diaphysis (immediately distal to the site of the Jones fracture zone). ${ }^{27-29}$ Differentiating an acute Jones fracture and other fracture types is clinically important because the watershed area at the metaphysis-diaphysis junction results in a higher risk of delayed union and nonunion of Jones fractures, compared to other fractures in this region (FIGURE 5). ${ }^{28,29}$

I Presentation. Proximal fifth-metatarsal fractures generally present with lateral foot pain and tenderness at the base of the fifth metatarsal, made worse by inversion of the foot, and inability to bear weight on the lateral aspect of the foot. Acute pain can follow a more insidious course of lateral foot pain in stress fracture.

IExamination. On exam, there might be swelling and ecchymosis over the lateral foot, with sharp tenderness to palpation at the base of the fifth metatarsal.

Imaging. Most fractures are revealed on standing AP, oblique, and lateral x-rays. Plain films are often falsely negative early in stress fracture; MRI is the gold standard of diagnosis. $^{27,30}$

I Management. Preferred treatment for

On a plain film of this injury, the distance between the base of the first metatarsal and the base of the second metatarsal (double-headed arrow) is $>2 \mathrm{~mm}$. Another clue (not shown) to a disturbance of the Lisfranc joint complex is an avulsion fracture at the medial base of the second metatarsal or distal lateral corner of medial cuneiform (the "fleck sign").

athlete can begin once the patient can walk down multiple flights of stairs without pain. ${ }^{24}$

Orthopedic referral for surgical evaluation is recommended for all patients who have any radiographic evidence of dynamic instability, indicated by the fleck sign; displacement; or obvious diastasis between the metatarsals on imaging. A delay of 1 to 2 weeks from injury to fixation has not been associated with a negative outcome; delay as long as 6 weeks is permissible in some cases. Longer delay in surgical treatment ( $\geq 6$ months) can be associated with posttraumatic arthritis and the need for Lisfranc fusion. ${ }^{24-26}$ a nondisplaced tuberosity avulsion fracture is typically 2-pronged: compressive dressings or casting for pain control and weightbearing and range-of-motion exercises as tolerated. ${ }^{1}$ Follow-up every 2 to 3 weeks is recommended to ensure appropriate healing-ie, pain nearly resolved by 3 weeks post-injury and radiographic union evident at 8 weeks. If displacement is $>3 \mathrm{~mm},>60 \%$ of the metatarsal-cuboid joint surface is affected, or there is a 1 to $2 \mathrm{~mm}$ step-off on the cuboid articular surface, consider referral to an orthopedist. ${ }^{1,29}$

Jones fractures can be managed initially with posterior splinting, non-weight-bearing, and close follow-up. When radiographic healing has not been achieved by 6 to 8 weeks, non-weight-bearing status can be extended by another 4 weeks. When displacement is $>2 \mathrm{~mm}$, or there is no healing after 12 weeks 


\section{FIGURE 5}

\section{Proximal fifth-metatarsal fractures}
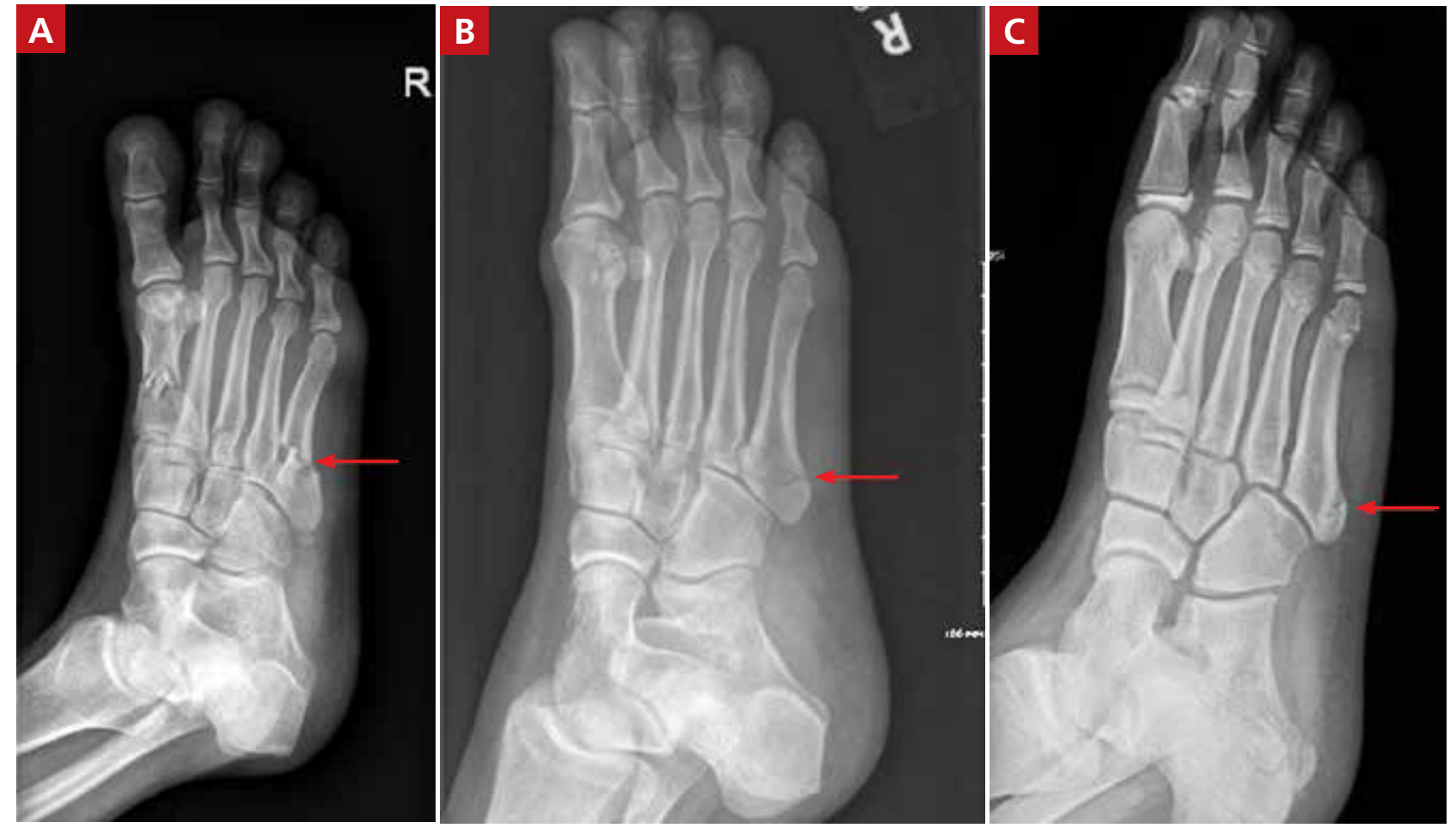

Shown here: Two variations of a Jones fracture (A and B) and an avulsion fracture of the tuberosity (C).

of immobilization and delayed union on $\mathrm{x}$-rays, referral for surgical management is indicated. ${ }^{1}$ In select cases, when earlier return to activity is desired, referral for early surgical fixation is appropriate. ${ }^{27}$

Surgical referral is indicated in all cases of diaphysial stress fracture because of the high rate of nonunion and refracture. Conservative management, based on the orthopedic surgeon's assessment, might be an option in a minority of patients. ${ }^{29}$

\section{CORRESPONDENCE}

Aileen Roman, MD, Boston University Medical School,

Department of Family Medicine, 11 Melnea Cass Boulevard, Boston MA, 02119; aileen.roman@bmc.org

\section{References}

1. Bica D, Sprouse RA, Armen J. Diagnosis and management of common foot fractures. Am Fam Physician. 2016;93:183-191.

2. Gross CE, Nunley JA 2nd. Acute Achilles tendon ruptures. Foot Ankle Int. 2016;37:233-239.

3. Cooper MT. Acute Achilles tendon ruptures: does surgery offer superior results (and other confusing issues)? Clin Sports Med. 2015;34:595-606.

4. Maffulli N, Via AG, Oliva F. Chronic Achilles tendon disorders: tendinopathy and chronic rupture. Clin Sports Med. 2015;34:607-624.
5. Hutchison A-M, Evans R, Bodger O, et al. What is the best clinica test for Achilles tendinopathy? Foot Ankle Surg. 2013;19:112-117.

6. Kadakia AR, Dekker RG2nd, Ho BS. Acute Achilles tendon ruptures: an update on treatment. Am Acad Orthop Surg. 2017;25:23-31.

7. van Zuuren WJ, Schepers T, Beumer A, et al. Acute syndesmotic instability in ankle fractures: a review. Foot Ankle Surg. 2017;23:135-141.

8. van Dijk CN, Longo UG, Loppini M, et al. Classification and diagnosis of acute isolated syndesmotic injuries: ESSKA-AFAS consensus and guidelines. Knee Surg Sports Traumatol Arthrosc. 2016;24:1200-1216.

9. Fort NM, Aiyer AA, Kaplan JR, et al. Management of acute injuries of the tibiofibular syndesmosis. Eur J Orthop Surg Traumatol. 2017;27:449-459.

10. Miller TL, Skalak T. Evaluation and treatment recommendations for acute injuries to the ankle syndesmosis without associated fracture. Sports Med. 2014;44:179-188.

11. Hunt KJ, Phisitkul P, Pirolo J, et al. High ankle sprains and syndesmotic injuries in athletes. J Am Acad Orthop Surg. 2015;23: 661-673.

12. DeWeber K. Syndesmotic ankle injury (high ankle sprain). UpToDate. September 17, 2019. www.uptodate.com/contents/ syndesmotic-ankle-injury-high-ankle-sprain. Accessed May 26 2020.

13. Goost H, Wimmer MD, Barg A, et al. Fractures of the ankle joint: investigation and treatment options. Dtsch Arztebl Int 2014;111:377-388.

14. Qin C, Dekker RG, Helfrich MM, et al. Outpatient management of ankle fractures. Orthop Clin North Am. 2018;49:103-108.

15. Stiell IG, Greenberg GH, McKnight RD, et al. Decision rules for the use of radiography in acute ankle injuries. Refinement and prospective validation. JAMA. 1993;269:1127-1132.

16. Jenkin M, Sitler MR, Kelly JD. Clinical usefulness of the Ottawa Ankle Rules for detecting fractures of the ankle and midfoot. $J$ Athl Train. 2010;45:480-482. 
17. Glas AS, Pijnenburg BACM, Lijmer JG, et al Comparison of diagnostic decision rules and structured data collection in assessment of acute ankle injury. CMAJ. 2002;166:727-733.

18. Leduc S, Nault M-L, Rouleau DM, et al. My experience as a foot and ankle trauma surgeon in Montreal, Canada: what's not in the books. Foot Ankle Clin. 2016;21:297-334.

19. Ibrahim MS, Jordan R, Lotfi N, et al. Talar head fracture: a case report, systematic review and suggested algorithm of treatment. Foot (Edinb). 2015;25:258-264.

20. Shank JR, Benirschke SK, Swords MP. Treatment of peripheral talus fractures. Foot Ankle Clin. 2017;22:181-192.

21. Kwaadu KY. Management of talar fractures. Clin Podiatr Med Sur. 2018;35:161-173.

22. Yu JS. Easily missed fractures in the lower extremity. Radiol Clin North Am. 2015;53:737-755.

23. Welck MJ, Zinchenko R, Rudge B. Lisfranc injuries. Injury. 2015;46:536-541.
24. Seybold JD, Coetzee JC. Lisfranc injuries: when to observe, fix, or fuse. Clin Sports Med. 2015;34:705-723.

25. Puna RA, Tomlinson MPW. The role of percutaneous reduction and fixation of lisfranc injuries. Foot Ankle Clin. 2017;22:15-34.

26. Weatherford BM, Bohay DR, Anderson JG. Open reduction and internal fixation versus primary arthrodesis for Lisfranc injuries. Foot Ankle Clin. 2017;22:1-14.

27. Porter DA. Fifth metatarsal Jones fractures in the athlete. Foot Ankle Int. 2018;39:250-258.

28. Cheung CN, Lui TH. Proximal fifth metatarsal fractures: anatomy, classification, treatment and complications. Arch Trauma Res. 2016;5:e32298.

29. Alsobrook J, Hatch RL. Proximal fifth metatarsal fractures. UpToDate. January 31, 2020. www.uptodate.com/contents/proximalfifth-metatarsal-fractures. Accessed May 26, 2020.

30. Welck MJ, Hayes T, Pastides P, et al. Stress fractures of the foot and ankle. Injury. 2017;48:1722-1726.

\section{Access a special supplement, podcasts, and a patient handout on migraine management, including:}

- Treatment Patterns and Unmet Needs in the Acute Treatment of Migraine

- Pharmacology and Pharmacokinetics of Ubrogepant

- Clinical Efficacy and Safety of Ubrogepant for Acute Treatment of Migraine

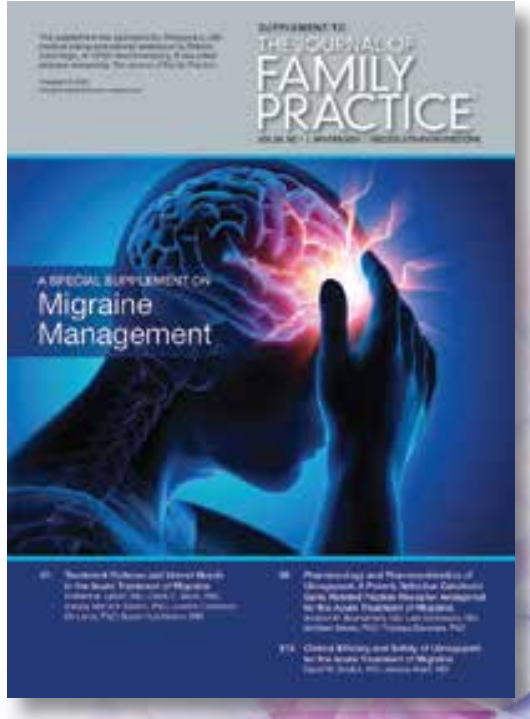

This supplement can be found at www.mdedge.com/MigraineManagement 